\title{
On the Riemann P Equation with Integral Exponent differences.
}

By W. L. Frrrar.

(Read 5th December 19:24. Received s0th January 1925.)

\section{INTRODUCTION.}

The differential equation in question is of the second order and has three regular singular points. It is usually denoted by

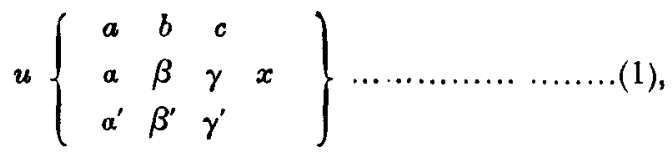

where $a, b, c$ are the singular points, $\alpha, \alpha^{\prime}$ etc. the exponents at those singularities. The solution when no one of the numbers $a-\alpha^{\prime}, \beta-\beta^{\prime}, \gamma-\gamma^{\prime}$ is an integer or zero is well known; all types of solution are expressible in terms of the hypergeometric functions.

On the other hand, when one of the above numbers is integral or zero only one solution is readily obtained and the theory of the second solution is somewhat involved. Thus, in Lindelöf's memoir on the subject (Acta Societ. Scient. Fennicoe XIX (1893) p. 15), the equation

$$
u\left\{\begin{array}{ccl}
0 & \infty & 1 \\
0 & a & 0 \\
1-\gamma & \beta & \gamma-\alpha-\beta
\end{array}\right\}
$$

is considered when $1-\gamma$ is a negative integer. The second solution, which together with $F(\alpha, \beta ; \gamma ; x)$ forms a fundamental system of integrals of the equation, is there given as

$$
x^{1-\gamma} G(\alpha, \beta ; \gamma ; x)+C . \log x . F(\alpha, \beta ; \gamma ; x),
$$

where $C$ is a certain constant and

$$
\begin{aligned}
& G(\alpha, \beta ; \gamma ; x)=1+\frac{(\alpha-\gamma+1)(\beta-\gamma+1)}{1(2-\gamma)} x .+\ldots \\
& +\left(\frac{a-\gamma+1)(\alpha-\gamma+2)-(\alpha-2)(\beta-\gamma+1)}{1.2 .3 \ldots(\gamma-2)(2-\gamma)(3-\gamma) \ldots(-2)(-1)} \frac{(\beta-\gamma+2) \ldots(\beta-2)}{1-2-2}\right. \\
& -C x^{\gamma-1}\left\{N+\left[N-\left(\frac{a+\beta}{a \cdot \beta}-\frac{\gamma+1}{1 \cdot \gamma}\right)\right] a_{1} x+\ldots\right\} \text {. } \\
& a_{n}=a(\alpha+1) \ldots(\alpha+n-1) \beta(\beta+1) \ldots(\beta+n-1) / \gamma(\gamma+1) \ldots(\gamma+n-1)(n !) \text {, } \\
& \text { while } N \text { is obtained as the sum of a certain infinite series. }
\end{aligned}
$$


This type of solution is difficult to handle, and introduces into the theory of the hypergeometric equation functions other than the well known hypergeometric series.

The present paper shews that the complete solution of $(1)$ is in all cases expressible in terms of hypergeometric series. The result of effecting the solution in the way here adopted is the introduction of generalised Legendre functions with properties analogous to $P_{n}(z)$ and $Q_{n}(z)$.

\section{\$1. Reduction of (1) to standard form.}

Suppose that $a^{\prime}-a$ is zero or an integer. Then a suitable $(x, z)$ transformation will change (1) into

$$
u\left\{\begin{array}{cccc}
0 & 1 & \infty & \\
a & \beta & \gamma & z \\
a^{\prime} & \beta^{\prime} & \gamma^{\prime} &
\end{array}\right\} .
$$

Let $\alpha^{\prime}-\alpha=r \geq 0, \beta^{\prime}-\beta=s, \gamma^{\prime}-\gamma=t$.

$$
\text { Write } \quad u=z^{a^{\prime}}(z-1)^{\beta^{\prime}} v \text {. }
$$

Then $v$ satisfies the equation given by the scheme

$$
v\left\{\begin{array}{rrl}
0 & 1 & \infty \\
0 & 0 & a^{\prime}+\beta^{\prime}+\gamma \\
-r & -8 & a^{\prime}+\beta^{\prime}+\gamma^{\prime}
\end{array} \quad z \quad\right. \text {. }
$$

If, further, we write $v=d^{r} w / d z^{r}, w$ satisfies the equation

$$
w\left\{\begin{array}{lll}
0 & 1 & \infty \\
0 & 0 & \alpha+\beta^{\prime}+\gamma \quad z \\
0 & r-8 & \alpha+\beta^{\prime}+\gamma^{\prime}
\end{array}\right\},
$$

or putting $\lambda=r-s,-n=\alpha+\beta^{\prime}+\gamma$,

$$
\begin{aligned}
& w\left\{\begin{array}{lll}
0 & 1 & \infty \\
0 & 0 & -n \\
0 & \lambda & n-\lambda+1
\end{array}\right\} . \\
& \text { Solutions of (1. c.) }
\end{aligned}
$$

\section{§2. 1. The first solution.}

The first solution is $F(-n, n-\lambda+1 ; 1 ; z)$ and its analytic continuations. Denote it by $P_{n, \lambda}(z)$.

This function may be defined by an integral of the type used to define $P_{n}(z)$ in Whittaker and Watson, Modern Analysis 
(3rd edition), p. 307, and the theory of $P_{n, \lambda}(z)$ is practically identical with that of $P_{n}\left(\frac{1-z}{2}\right)$, to which it reduces when $\lambda=0$.

The equation (1.c) may be written

and

$$
z(1-z) w^{\prime \prime}+\{1-z+(\lambda-1) z\} w^{\prime}+n(n-\lambda+1) w=0,
$$

$$
\frac{1}{2 \pi i} \int_{C} t^{n-\lambda}(1-t)^{n}(t-z)^{\cdots+\lambda-1} d t
$$

satisfies it if $C$ be so chosen that

$$
t^{n-\lambda+1}(1-t)^{n+1}(t-z)^{-n+\lambda-2}
$$

returns to its initial value after the description of the contour $C$.

Let $A$ be a point on the real axis between 0 and 1 in the $t$ plane, and if $z$ be real and $0<z<1$, to the right of $t=z$. Make the integrand definite by taking at $A$, from which $C$ starts, $|\arg (t-z)|<\pi, \arg t=0, \arg (1-t)=0$. Further let the $t$ and $z$ planes be cut from 1 to $\infty$ along the real axes, and in the cut $t$ plane let $C$ be a contour encircling the points $t=0$ and $t=z$ once each in a counter-clockwise direction. When $C$ is thus restricted denote it by $(A ; 0+, z+)$.

$$
\text { Then } P_{n, \lambda}(z)=\frac{1}{2 \pi i} \int_{A}^{(0+, z+)} t^{n-\lambda}(1-t)^{n}(t-z)^{-n+\lambda-1} d t \text {. }
$$

The hypergeometric form when $|z|<|\cdot 1-\delta|$ is obtained by taking $C$ to be $|t|=1-\delta, 0<\delta<1$. The work follows exactly on the lines of $\$ 15.22$ Whittaker and Watson, loc. cit.

The analogues of $P_{n}(z)=\frac{1}{2^{n} \cdot n !} \frac{d^{n}}{d z^{n}}\left(z^{2}-1\right)^{n}$ when $n$ is an integer, are obtained as particular cases of Jacobi's polynomials. ${ }^{*}$ If both $n$ and $\lambda$ are positive integers, $n \geq \lambda$, the integral definition gives

$$
P_{n, \lambda}(z)=\frac{1}{(n-\lambda) !} \frac{d^{n-\lambda}}{d z^{n-\lambda}}\left\{z^{n-\lambda}(1-z)^{n}\right.
$$

In this form it is obtained by Jacobi's formula for

$$
(1-z)^{\lambda} F(n+1,-n+\lambda ; 1 ; z) \text {, }
$$

which is obtained from $F(-n, n-\lambda+1 ; 1 ; z)$ by Euler's transformation. $t$

(a) Gesammelte Werke VI, 184-202.

(b) Journal für Math. 56 (1859), 149-165.

$+F(a, b ; c ; x)=(1-x)^{c-a-b} F(c-a, c-b ; c ; x)$. 
\$2.2. The second solution.

If $n, \lambda$ are positive integers, $n \geq \lambda$, and $z$ is not a real number lying between 0 and 1 , let

$$
R_{n, \lambda}(z)=\int_{0}^{1}\left\{P_{n, \lambda}(y) /(y-z)\right\} d y .
$$

Then $(n-\lambda) ! R_{n, \lambda}(z)=\int_{0}^{1} \frac{d^{n-\lambda}}{d y^{n-\lambda}}\left\{y^{n-\lambda}(1-y)^{n}\right\} \frac{d y}{y-z}$

or, integrating by parts,

$$
R_{n, \lambda}(z)=(-1)^{n-\lambda+1} \int_{0}^{1} y^{n-\lambda}(1-y)^{n}(z-y)^{-n+\lambda-1} d y .
$$

If $|z|>1,(1-y / z)^{-n+\lambda-1}$ may be expanded by the binomial theorem when $y$ lies in the range $(0,1)$, and the R.H.S. of the last equation reduced to the form

$$
\begin{aligned}
(-z)^{-n+\lambda-1} \frac{\Gamma(n-\lambda+1) \Gamma(n+1)}{\Gamma(2 n-\lambda+2)} & \\
& H\left(n-\lambda+1, n-\lambda+1 ; 2 n-\lambda+2 ; \frac{1}{z}\right) .
\end{aligned}
$$

Now it is well known that $F\left(a, b ; c ; \frac{1}{y}\right)$ is a solution of

$$
P\left\{\begin{array}{ccc}
0 & 1 & \infty \\
a & 0 & 0 \\
b & c-a-b & 1-c
\end{array} \quad y\right\} .
$$

Using this fact it is easy to show that (4) is a solution of (1.c).

The solution in the general case may therefore be taken to be the expression (4) and its analytical continuations. By using the known theory of the hypergeometric function we may define $R_{n, \lambda}(z)$ as

$(-z)^{-n+\lambda-1} \frac{\Gamma(n+1)}{\Gamma(n-\lambda+1} \frac{1}{2 \pi i} \int_{-\infty i}^{\infty i} \frac{\{\Gamma(n-\lambda+s+1)\}^{2} \Gamma(-s)}{\Gamma(2 n-\lambda+s-2)}\left(-\frac{1}{z}\right)^{d} d s$,

the path of integration being taken so that the poles of $\Gamma(n-\lambda+8+1)$ lie on the left of the path and those of $\Gamma(-8)$ on the right of it (cf. Whittaker and Watson, loc. cit. $\S 14.5)$.

\$2.21. Exceptional cases.

If $2 n-\lambda+2$ is zero or a negative integer the hypergeometric series in its usual form and theory is not applicable If, however, we write in $(1, c) w=(1-z)^{\wedge} \theta, \theta$ satisfies

$$
\theta\left\{\begin{array}{rrr}
0 & 1 & \infty \\
0 & -\lambda & -n+\lambda \\
0 & 0 & n+1
\end{array} \quad z\right\}
$$


Let $N=-(n+1), L=-\lambda$. Then the solutions of the lattel equation are $P_{N, L}(z)$ and $k_{N, L}(z)$ while $2 N-L+2=-2 n+\lambda$ is a positive integer. We may therefore confine our attention to forms of $(1 . c)$ in which $2 n-\lambda+2$ is neither zero nor a negative integer.

The numerical factor contains infinities if $n-\lambda+1$ or $n+1$ is zero or a negative integer. If $n-\lambda+1=-m$ ( $m$ positive integer), the equation $(1, c)$ is unaltered when we write $m$ for $n$, and we may take $P_{m, \lambda}(z), R_{m, \lambda}(z)$ as the solutions. This still leaves a difficulty when $n$ is itself a positive integer and $\lambda$ a positive integer greater than $n$. A similar difticulty remains when the case $n+1$ a negative integer is discussed. In both cases it is only the numerical factor that presents any difficulty: a second solution in the form of a hypergeometric function is obtainable. If $n-\lambda+1$ is zero or a negative integer (4) has only a finite number of terms and defines the solution everywhere, $(5)$ being then unnecessary.

\section{\$2.22. Comparison with $Q_{n}(z)$.}

The method of $\$ 2.2$ is that of Neumann for obtaining $Q_{n}(\dot{z})$ when $n$ is an integer. Moreover, if $|1-z|>2$,

$$
\begin{aligned}
R_{n, 0}\left(\frac{1-z}{2}\right)=\frac{\{\Gamma(n+1)\}^{2}}{\Gamma(2 n+2)}\left(\frac{z-1}{2}\right)^{-n-1} & \\
& F\left(n+1, n+1 ; 2 n+2 ; \frac{2}{1-z}\right),
\end{aligned}
$$

a form given by Barnes * for $Q_{n}(z)$.

In passing, it may be worthy of remark that, although the form (6) corresponds naturally to Murphy's expression for $P_{n}(z)$, and is moreover essily obtained from the definitions of $Q_{n}(z)$ usually adopted, e.g. $15.31,15.34$ Whittaker and Watson, loc. cit., this particular expression for $Q_{n}(z)$ is not generally used, and prominence is given to the non-symmetrical form with argument $1 / z^{2}$.

$$
\text { Solutions of }(1, b) \text {. }
$$

\section{\$3.1. The first solution.}

The solution of $(1, b)$ corresponding to $P_{n, \lambda}(z)$ is $\dagger$

$$
B_{n, \lambda}^{r}(z)=\frac{d^{r}}{d z^{r}} P_{n, \lambda}(z)
$$

* Quart. J. of Math. 39 (1908) 107.

+ The notation $P_{n, \lambda}^{r}$ is best reserved for the functions of $\$ 4$ which, for $\lambda=0$, reduce to assooiated Logendre Functions. 
a form which is valid save when $n$ is a positive integer less than $r$. When $|z|<1$, and $r-n$, if integral, is not negative, this may be written as

$$
\begin{aligned}
& \frac{d^{r}}{d z^{r}} F(-n n-\lambda+1 ; 1 ; z) \\
& =(-1)^{r} \frac{\Gamma(n+1) \Gamma(n-\lambda+r+1)}{\Gamma(r+1) \Gamma(n-r+1) \Gamma(n-\lambda+1)} \\
& \quad F(-n+r, n-\lambda+r+1 ; r+1 ; z) .
\end{aligned}
$$

This solution is, however, better regarded as one of the series (namely that valid for $|z|<1-\delta$ ) which may express the analytic function

$$
\frac{\Gamma(n-\lambda+r+1)}{\Gamma(n-\lambda+1} \frac{1}{2 \pi i} \int_{A}^{(0+,-z+)} t^{n-\lambda}(1-t)^{n}(t-z)^{-n+\lambda-r-1} d t,
$$

the procedure of $\$ 2.1$ being followed throughout.

$$
\text { Here } \quad t^{n-\lambda+1}(1-t)^{n+1}(t-z)^{-n+1-\lambda-r-2}
$$

must return to its initial value after $t$ has described the contour; this condition is satisfied whenever $v$ is an integer, positive or negative.

\section{$\$ 3.2$. The second solution.}

If $n, \lambda, r$ are positive integers, $n \geq \lambda, r$, and $z$ is not a real number lying in the interval $(0,1)$, let

$$
R_{n, \lambda}^{r,}(z)=r ! \int_{0}^{1}\left\{P_{n, \lambda}(y) /(y-z)^{r+1}\right\} d y .
$$

This, by the method of $\$ 2.2$ becomes

$$
\frac{\Gamma(n-\lambda+r+1)}{\Gamma(n-\lambda+1)} \int_{v}^{1} \frac{y^{n-\lambda}(1-y)^{n}}{(y-z)^{n-\lambda+r+1}} d y
$$

or, when $|z|>1$,

$$
\begin{aligned}
& (-z)^{-n+\lambda-i-1} \frac{\Gamma(n-\lambda+r+1) \Gamma(n+1)}{\Gamma(2 n-\lambda+2)} \\
& \quad F\left(n-\lambda+r+1, n-\lambda+1 ; 2 n-\lambda+2 ; \frac{1}{z}\right) .
\end{aligned}
$$

The definition is, in essence, $d^{r} / d z^{r}\left\{l_{n, \lambda}(z)\right\}$. Its extension to the general case may be effected by the procedure used for $R_{n, \lambda}(z)$ in $\S 2.2$, and the exceptional cases dealt with as in $\$ 2.21$. 
\$4. The appearance of these functions in other work.

(a) The function of most interest associated with $B_{n, \lambda}{ }^{r}(z)$ is

$$
P_{n, \lambda}^{r}(z)=z^{r / 2}(1-z)^{(r-\lambda) / 2} B_{n, \lambda}^{r}(z),
$$

considered by Bateman, Proc. Lond. Math. Soc. (2) 3 (1904-5) 111-123. It there arises from a discussion of the differential equation

$$
\frac{\partial^{2} V}{\partial x_{1}^{2}}+\frac{\partial^{2} V}{\partial x_{2}^{2}}+\frac{\partial^{2} V}{\partial x_{3}^{2}}+\frac{\partial^{2} V}{\partial x_{4}^{2}}=0
$$

The notations are connected thus: $-v=(n+k-m) / 2, \lambda=k-m$, $r=k, z=(1-\mu) / 2$,

The function

$$
P_{\nu, \lambda}^{\nu}(z)=(-1)^{r} \frac{\Gamma(\nu+1)}{\Gamma(\nu-\lambda+1)} \Theta_{n}{ }^{m, k}(\mu) .
$$

$$
Q_{v, \lambda}^{r}(z)=z^{r / 2}(1-z)^{(r-\lambda) / 2} R_{v, \lambda}^{r}(z)
$$

is the second solution of the hypergeometric equation given by Bateman on page 112,* when this is expressed in terms of $v, \lambda, r, z$.

(b). The polynomials $A_{n}{ }^{\prime}(x)$ given by Richardson, Quart. J. of Math. 50 (1923) 89-96, as bi-orthogonal to $x^{*}$ over $(0,1)$ are expressed rather curiously as

$$
-\frac{1}{(t !)^{2}} \frac{d^{t}}{d x^{t}}\left\{x^{t}(1-x)^{t} B_{n+1,1}^{t+1}(x)\right\}
$$

The equivalence of the two functions is obtained by writing

$$
\begin{aligned}
B_{n+1.2}^{t+1}(x) & =(-1)^{t+1} \frac{\Gamma(n+2) \Gamma(n+t+2)}{\Gamma(t+2) \Gamma(n-t+1) \Gamma(n+1)} \\
& F(-n+t, n+t+2 ; t+2, x) \\
& =(-1)^{t+1} \frac{(n+1) \Gamma(n+t+2)}{\Gamma(t+2) \Gamma(n-t+1)}(1-x)^{-t} F(n+2,-n ; t+2 ; x),
\end{aligned}
$$

using Euler's transformation.

(c) When $n$ is a positive integer, or whenever the hypergeometric forms reduce to polynomials, the functions are particular cases of the Jacobi polynomials. The properties of these, reeurrence formulae, order when $n$ is large, analogue of Heine's expansion for $1 /(t-z)$ etc. are dealt with by Darboux, Journal de Math (3) 4 (1878) $1-56,377-416$. If we write $Q_{n, \lambda}(x)=(1-x)^{-\lambda} R_{n, \lambda}(x)$, then by using Euler's transformation of the form (4), we see that $Q_{n, \lambda}(x)$ is the $Q$ function of Darboux (p. 400 equation (41)) when $\alpha=1-\lambda, \gamma=1$.

* When $\lambda=0$, these functions become the associated Legendre functions, 
\$5. Orthogonal and integral properties when $n, \lambda$ are integers, $n \geq \lambda$.

(a) $B_{n,-k-k}(x)$, when $k \leq s$, is a numerical multiple of

$$
F_{n}(x)=(-1)^{n} \frac{(n+k+1) !}{k !(n !)^{2}} \frac{d^{n}}{d x^{n}}\left\{x^{n+k-1}(1-x)^{n}\right\} .
$$

Also, $\int_{0}^{1} x^{2} F_{n}(x)=0$ or 1 according as $8<$ or $=n$. The roots of $F_{n}(x)$ are all different and lie between 0 and 1 . The theory of this function is simpler than that of $A_{n}{ }^{2}(x)$ given in $\S 4(b)$.

(b) $A$ curious result in this direction is that if

$$
F_{n, t}(x)=x^{1-\lambda} \frac{d^{t}}{d x^{t}}\left\{x^{t+\lambda-1}(1-x)^{t} B_{n+\lambda, \lambda}^{t+\lambda}(x)\right\}, n \geq \lambda, t,
$$

and $\lambda<s+2$,

$$
\begin{aligned}
\int_{0}^{1} x^{t} F_{n, t}^{\prime}(x) d x & =(-1)^{\lambda} t !(t+\lambda-1) ! \text { when } s=t+\lambda-1, \\
& =0 \text { when } s \neq t+\lambda-1, \text { but remains } \leq n+\lambda-1 .
\end{aligned}
$$

It may be noted that the limitation $\lambda<s+2$ makes $\lambda=1$, the case of Richardson's polynomials, the only example of this type which serves to construct a series of functions bi-orthogonal to $1, x, x^{2}, \ldots$ respectively over $(0,1)$.

(c) $m, n, \lambda$ being positive integers, $m-n \geq \lambda, n \geq \lambda$,

$$
\begin{aligned}
\int_{0}^{1} P_{m, \lambda}(z) P_{n, \lambda}(z) d z & =0 \quad \text { when } m-n>\lambda, \\
& =\frac{(2 n-\lambda) !(n+\lambda) !}{(2 n+\lambda+1) !(n-\lambda) !} \text { when } m-n=\lambda .
\end{aligned}
$$

Integration by parts is sufficient to establish the result.

(d) $m, n, \lambda$ positive integers; $m \geq n \geq \lambda \geq 0$.

$$
\begin{aligned}
\int_{0}^{1}(1-z)^{-\lambda} P_{m, \lambda}(z) P_{n, \lambda}(z) d z & =0 \quad \text { when } m \neq n, \\
& =1 /(2 n-\lambda+1) \text { when } m=n .
\end{aligned}
$$

This is merely a transcription of a known property of Jacobi polynomials (compare Jacobi, loc. cil. $\$$ ).

(c) $m, n, \lambda, r$ positive integers; $m \geq n \geq \lambda, r$.

$$
\begin{array}{rlrl}
\int_{0}^{1} P_{n, \lambda}^{r}(z) P_{m, \lambda}^{r}(z) d z & =0 & \text { when } m \neq n, \\
& =\frac{n !(n-\lambda+r) !}{(n-r) !(n-\lambda) !} \frac{1}{2 n-\lambda+1} \text { when } m=n .
\end{array}
$$


The proof when $r \geq \lambda$ is merely that of Jacobi (cited above), when we begin his method from the equation satisfied by $F(-n+r, n-\lambda+r+1 ; r+1 ; z)$, written in the prepared form,

$$
\frac{d}{d z}\left\{y^{\prime} z^{r+1}(1-z)^{r+1-\lambda}\right\}=-(n-r)(n-\lambda+r+1) y z^{\prime \prime}(1-z) .^{r-\lambda} .
$$

This proof requires $r+1$ and $r+1-\lambda$ to be positive.

If $r<\lambda$ a less direct proof is necessary. If $Y_{n}$ denote $F(-n+r, n-\lambda+r+1 ; r+1 ; z)$, then for $r<\lambda \leq n$,

$$
Y_{n}=(1-z)^{\lambda-r} F(n+1, \lambda-n ; r+1 ; z)
$$

is a polynomial. Moreover, the $k^{\text {th }}$ derivative of $Y_{n}$, say $Y_{n}^{(k)}$, contains $(1-z)^{\lambda-r-k}$ as a factor if $k \leq \lambda-r$. The method may now dispense with the condition $r+1-\lambda$ positive and gives

$$
\int_{0}^{1} z^{r}(1-z)^{r-\lambda} Y_{m} Y_{n} d z=N \int_{0}^{1} z^{\lambda} Y_{m}^{(\lambda-r)} Y_{n}^{(\lambda-r)} d z,
$$

where $N$ is a numerical factor.

The final result is obtained by recommencing the method with the equation satisfied by $Y_{n}^{(\lambda-r)}$ i.e.

$$
\frac{d}{d z}\left\{y^{\prime} z^{\lambda+1}(1-z)\right\}=-(n-\lambda)(n+1) z^{\lambda} y .
$$

